\title{
Changes in the mechanical properties of femoral cartilage tissue in advanced osteoarthritis
}

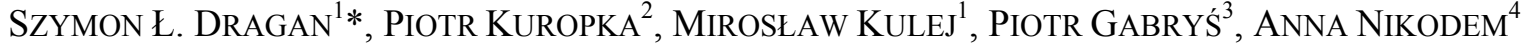 \\ ${ }^{1}$ Department of Regenerative and Restoration Medicine in Orthopedics, Department of Orthopaedics and Traumatology, \\ Wrocław Medical University, Wrocław, Poland. \\ ${ }^{2}$ Division of Histology and Embryology, Wrocław University of Environmental and Life Sciences, Wrocław, Poland. \\ ${ }^{3}$ Clinic of Orthopaedics and Traumatology, Wrocław University Hospital, Wrocław, Poland. \\ ${ }^{4}$ Department of Mechanics, Materials and Biomedical Engineering, Faculty of Mechanical Engineering, \\ Wrocław University of Science and Technology, Wrocław, Poland.
}

\begin{abstract}
Purpose: The main goal of this article was the analysis of the changes of mechanical properties, thickness and histology of the cartilage in different regions of the femur head in advanced stage of the osteoarthritis. Methods: The study material consisted of cylindrical specimens $(9.7 \mathrm{~mm})$ prepared form proximal epiphysis of the human femur bone after hip arthroplasty. The thickness was determined from the reconstruction of the specimen from microtomography (SkyScan 1172, Bruker ${ }^{\mathbb{B}}$ ) images. Mechanical properties of the cartilage tissue were identified in static indentation test conducted the use of MTS ${ }^{\circledR}$ Synergie 100 testing machine. Histological study enabled us to determine cartilage total thickness as well as location and orientation of the collagen fibers. Results: Depending on the region of the head, the cartilage tissue thickness was in the range $(0.74-2.23 \mathrm{~mm})$. The lowest thickness values were obtained for the R5 region and the lowest for R4. Samples from R1-R4 regions differ by 24\%. Measurements of cartilage tissue indentation, determined with the help of Hayes' formula, showed that the values of mechanical parameters in regions R1, R2, R3 and R5 have similar values (the difference between them is about 5.5\%). Region R4, which had the smallest thickness, also had the lowest values of mechanical parameters. Conclusions: Using $\kappa$ coefficients, proposed by Hayes, enabled us to obtain similar values of mechanical parameters in the regions R1, $\mathrm{R} 2$, R3 and R5, despite differences in cartilage tissue thickness. The R4 region, which had the weakest mechanical parameters, was characterized by the strongest reduction of the articular cartilage tissue, which was accompanied by an unformed mass of cartilage residue originating from the abraded surface and bursa fluid.
\end{abstract}

Key words: cartilage tissue, osteoarthritis, indentation test, histology, mechanical properties

\section{Introduction}

Osteoarthritis (OA) is a chronic and incurable disease affecting diarthrodial joints. It is one of the ten most frequent diseases affecting bone and joint system and one of the most frequent medical issues affecting humans becoming important socio-economic challenge. According to Osteoporotic Fractures Research Group [16], 7.2\% of women above 65 years of age suffer from OA. Similar research from Johnston County OA Project show that OA affects as much as
$27 \%$ of people aged 45 and above. Osteoarthritis reduces mobility of the joints but affects also the whole kinematic chain. It leads to degeneration of joint cartilage that is characterized by an extracellular matrix damage and an significant loss in tissue cellularity [28], narrowing of the joint gap, osteophytes, and cysts development leading to subchondral sclerosis [12]. Changes to the mechanical properties, including strength and load bearing capabilities in hip, are result of biological, biochemical and metabolic processes ongoing in tissues affected with OA [9]. As the disease progress, cartilage layer degenerates gradually,

\footnotetext{
* Corresponding author: Szymon Ł. Dragan, Wrocław Medical University, ul. Borowska 213, 50-556, Wrocław, Poland. Phone: 48 662555565, e-mail: dragan.szymon@gmail.com

Received: September 28th, 2019

Accepted for publication: February 12th, 2020
} 
reducing cartilage shock absorbing and load bearing properties, and changes load distribution compared to healthy joint. This negative effect is not limited to the joint. As a result of cartilage destruction, the load between pelvis and femur increases. This is clearly observed in 2nd and 3rd stage (Beck and Outbrige classification [8], [18]) of the OA when the remodeling processes start and lead to increased density of bone tissue in regions that bear the highest load. If the OA progresses, also the geometry of joint changes. The aforementioned changes are often visible in 3rd stage of the disease when there is a clear increase in the density, especially in subchondral layer. Changes to the femur head and acetabulum are not evenly distributed but depend on the thickness of the subchondral and its properties. These, in turn, depend on the number and distribution of the collagen fibers and aggregating proteoglycans in extracellular matrix (ECM).

Typical treatment in this stage includes total hip arthroplasty which is considered the biggest achievement of the orthopedics. Ageing of the society, increasing expected lifetime and patient expectations regarding the quality of life lead to the increase of the number of these surgeries [22]. Cartilage is a viscoelastic material. Viscoelasticity is related to intermolecular friction that is specific to polymers (including collagen) and materials having high water content ( $\sim 75-80 \%$ by wet weight), being shape compliance. Placing the cartilage for several hours under constant compressive load enables the researcher to determine the quasi-static compressive module at the level of 0.4-1.5 MPa [14]. Under joint movement, the cartilage absorbs and releases synovial fluid behaving similarly to a sponge and, therefore, is classified as a poroviscoelastic solid material.

Mechanical properties can be determined during indentation test where the indenter is introduced into the sample to a predefined depth while the force is measured. Originally, it was used to determine properties of homogenous durable materials and then expanded to polymers. Nowadays, this method is applied to measurements of hard tissues such as bone [3], [24], [29], calcium deposits [10] and highly deformable materials including articular cartilage [15], [26] and skin together with the subcutaneous tissues [2], [25]. Determination of mechanical properties of cartilage (AC) is not straightforward due to specific properties of the tissue and number of factors that affect the measurement. These include the articulating surfaces geometry, joint contact force, tissue material properties and the cartilage thickness which is related to the stiffness of the tissue. Moreover, the articular cartilage is inhomogeneous mechanically and in composition over its depth [11] that varies between 0.5 and $5.0 \mathrm{~mm}$. An early improvement to measurements was to take into consideration the tissue thickness assuming an incompressible condition and including an geometric factor that was determined experimentally.

The theoretical solution to the problem associated with axisymmetric indentation was found by Hayes et al. [5], who discovered that articular cartilage connected to subchondral bone was modelled as an elastic layer fixed to a rigid boundary.

Due to significant changes to the cartilage in the OA of femur, there are significant differences to cartilage thickness [13]. Differences are a consequence of cartilage loss in the areas of the highest load and tissue remodeling leading to hyperplasia in areas where load is small. The changes are not only quantitative (differences in the tissue thickness) but also qualitative, including different architecture of fibrous network of collagen (primarily type II) and changes in the elastic properties [4]. The main goal of this article is the analysis of the changes of mechanical properties and thickness of the cartilage in different regions of the femur head in advanced stage of the osteoarthritis.

\section{Materials and methods}

\subsection{Sample preparation}

The subject of the studies was the proximal epiphysis of the human femur bone after hip arthroplasty. Indication to conduct such treatment was advanced osteoarthritis of hip joint (stage 3 in Outerbrige and Beck scale). The surgeries were conducted at Department and Clinic of Orthopaedic and Traumatologic Surgery of the Wrocław Medical University and were approved by the Bioethics Committee of the Wrocław Medical University (approval no. $86 / 2010$ ). Samples included right femur heads from 5 females aged 56-64 and weighting $68-85 \mathrm{~kg}$. In all cases, no other autoimmunological diseases were identified that could have direct impact on the parameters investigated. Before the examination, samples were stored in hermetic plastic containers in temperature of $-20{ }^{\circ} \mathrm{C}$.

Because of the changes at the femur heads, including thickness and architecture of the cartilage, the experiments were conducted in 5 regions (R1-R5). The regions were selected so that the difference in the 

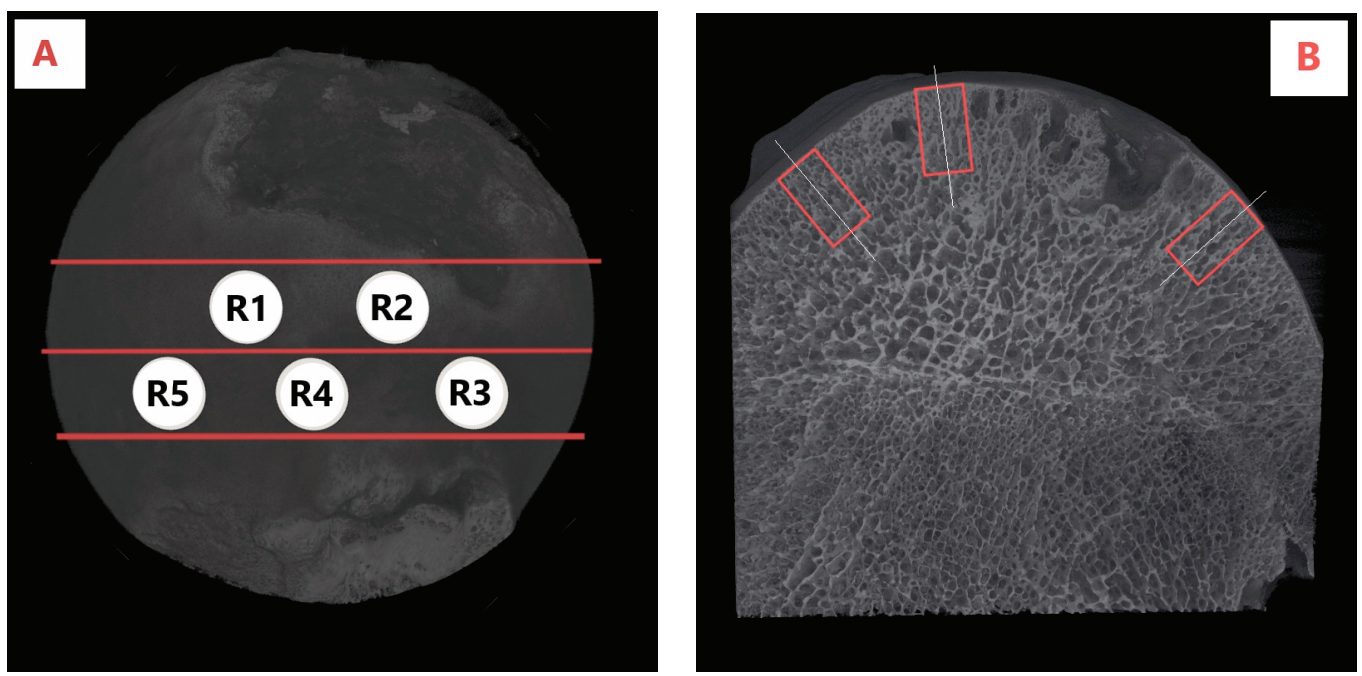

Fig. 1. A) The cylindrical bone-cartilage specimens were machined from different regions R1-R5 (10 mm), for indentation test and for histology (red cross-section); B) Example of central cross-section for patient with OA

tissue thickness (Resulting from the OA) was maximized and the remaining amount of cartilage was enough to conduct indentation. The regions also followed anatomic differentiation: R1 was located in superior area, R2 and R3 in posterior area, R4 in inferior area and R5 in anterior area. Cylindrical specimens were machined from selected locations with a core drill Falcon Trephine bur RA $\varnothing 10 \mathrm{~mm}$. The resulting specimen size was equal to $9.7 \mathrm{~mm}$. The axes of the specimens were as much as possible parallel to the long axis of the femur. The samples were then stored at $4{ }^{\circ} \mathrm{C}$ in a solution of isotonic phosphate buffered saline (PBS) until the mechanical test.

\subsection{Histology}

Histological evaluation was conducted for three $0.5 \mathrm{~mm}$ thick intersections prepared in the frontal plane in direct contact of the R1-R5 regions (Fig. 1A). Histological and histomorphometric analysis was conducted on cartilage samples from the top of the head and side surfaces. Investigations were conducted in polarized and fluorescent light, magnification 40 times and using UV-2A filter with following parameters: EX 330-380, DM 400, BA 420. The images captured using Nikon ECLIPSE 80i microscope were analyzed in NIS-Elements AR software. Stereoscopic techniques of image composition accompanied with morphometric techniques were used in investigations. This enabled the authors to determine cartilage total thickness as well as thickness of its layers. Based on the measurements, location and orientation of the collagen fibers were reconstructed.

\subsection{Cartilage thickness}

The thickness was determined from the reconstruction of the specimen from microtomography $(\mu \mathrm{CT})$ images. Images were acquired using $\mu \mathrm{CT}$ SkyScan 1172 , Bruker with resolution of $11 \mu \mathrm{m}$, voltage $59 \mathrm{kV}$ at $169 \mu \mathrm{A}$, and the thickness was measured in CTAn software. To prevent dehydration of the specimen during the scanning procedure, every specimen was secured with humidity chamber made out of a paper coating soaked with PBS fluid.

\subsection{Mechanical testing}

Mechanical cartilage tissue properties were identified in static indentation test conducted with MTS Synergie 100 testing machine with $500 \mathrm{~N}$ load cell. The mechanical articular cartilage and the underlying trabecular bone function is strongly influenced by the attachment to the subchondral bone plate. Therefore, simultaneous measurement of the mechanical properties of these two tissues without separation should be conducted. This way, the cartilage and the trabecular bone will be loaded equally during the mechanical testing, simulating the physiological conditions at the cartilage [18]. Indentation tests were carried with preload of $1 \mathrm{~N}$ to reduce timedepend effect on the resulting data [23], and low constant speed of $1 \mathrm{~mm}$ per minute. Due to significant influence of the friction during the test [30] the hemispherical indenter with radius $1.5 \mathrm{~mm}$ was used in the experiments. 
In contrast to classical stiffness test, the indentation test determines force equal to the resistance imposed by the sample under the strain. This defines the stiffness of the sample, which describes deformation of the sample under the influence of the force inelastic range (Fig. 6A). As a result, with increased stiffness and hardness of the specimen, the higher force is measured. Stiffness can be determined in a static and dynamic tests and the measured values depend significantly on the internal structure of specimen.

The amount of water in cartilage is one of the factors that heavily affects the measured stiffness. Precisely, the modulus of the material decreases linearly with increasing water content. Therefore, during the measurements, cartilage samples were hydrated with PBS solution. Based on the measurements, the indentation force-depth characteristics was determined and the force for 0.19 and $0.25 \mathrm{~mm}$ indentation was determined. The value of $0.19 \mathrm{~mm}$ correspond to $25 \%$ of maximal indentation. Based on these measurements, elastic modulus (1), shear modulus (2) and bulk modulus (3) for the indentation test of the cartilage tissue were calculated using relationships proposed by Hayes et al. [5].

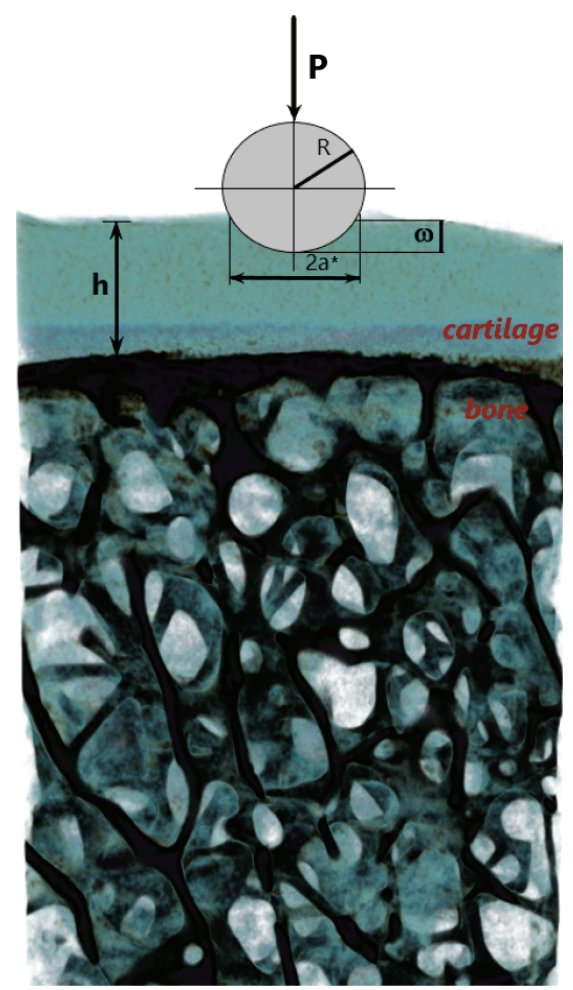

Fig. 2. Geometrical parameters of spherical indenter used in indentation tests

$$
E=\frac{P\left(1-v^{2}\right)}{2 a^{*} \omega \kappa}
$$

$$
\begin{gathered}
G=\frac{P(1-v)}{4 a^{*} \omega \kappa} \\
K=\frac{2 G(1-v)}{3(1-2 v)}
\end{gathered}
$$

where:

$E$ - instantaneous elastic modulus [MPa],

$G$ - shear modulus [MPa],

$K$ - bulk modulus [MPa],

$v$ - Poisson ratio,

$a$-radius of indenter [mm],

$a^{*}-$ polar radius $[\mathrm{mm}]$,

$\omega$ - indentation depth [mm],

$\kappa$ - scale factor [5] $a / h, v$.

Coefficient $\kappa$ in equations (1)-(2) depends on the shape of the indenter and was determined for several ratios of the indenter effective radius $a$ to tissue thickness $h$, and the Poissons's ratios $n$. The $a / h$ ratio represents geometrical and material-dependent factor, takes into account the change in the thickness of the cartilage as the indentation progress. Assuming that cartilage quality is constant, the thickness of the tissue does not affect the mechanical properties including elastic modulus [17], [27].

Assuming the cartilage is an incompressible material its Poisson's ratio equals to $v=0.45$ [6]. Indenter's effective hemispheric radius $a^{*}$ for a given indentation depth $\omega$ equals $a^{*}=\sqrt{(R-\omega)^{2}+R^{2}}$, where $R$ denotes the indenter radius (Fig. 2). Indenter is composed of chromium steel AISI 52100 (DIN 100CR6) with hardness equal to $60 \mathrm{HRC} / 700 \mathrm{HV}$.

\subsection{Statistical analysis}

Statistical analysis included basic characteristics, verification of normal distribution in the specimen (the Shapiro-Wilk test), analysis of the one-way variance (ANOVA). Statistical analysis was conducted in Origin 8.0 software with a statistical significance level of 0.05 .

\section{Results}

\subsection{Cartilage thickness}

Cartilage measurements were conducted based on tridimensional reconstructions of the specimen from microtomographic images (Fig. 3). 

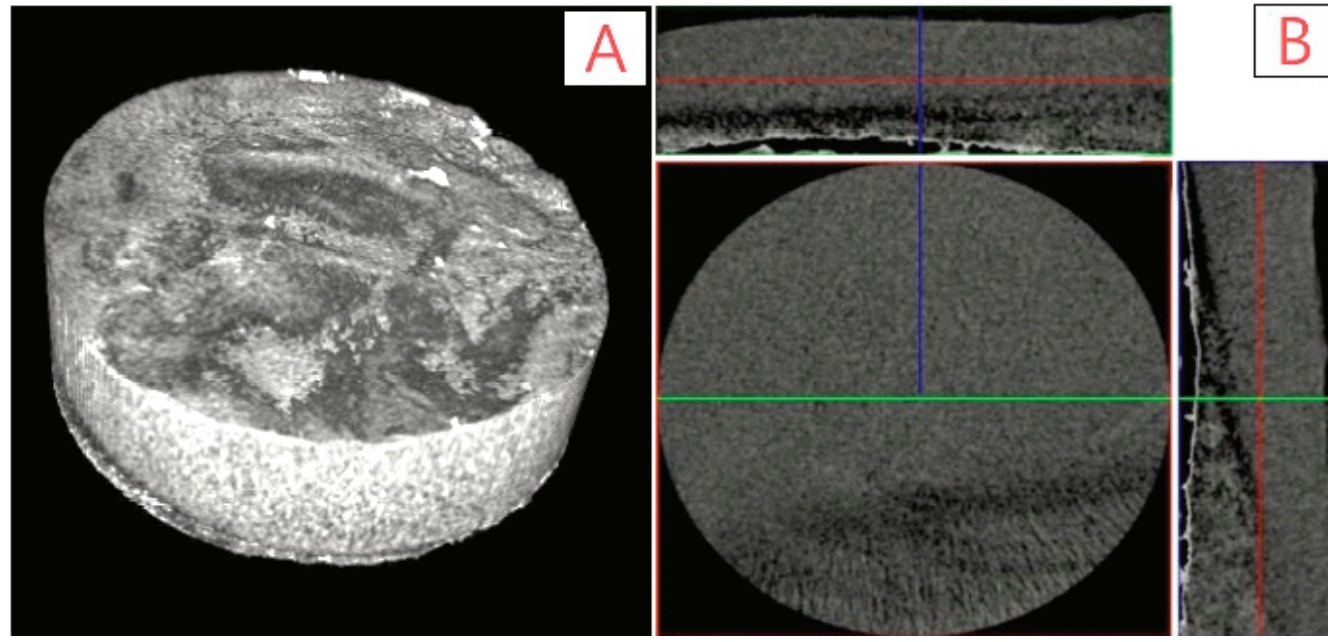

Fig. 3. Example of cartilage reconstruction from uCT images, CTVox (A) and DataViever (B) software
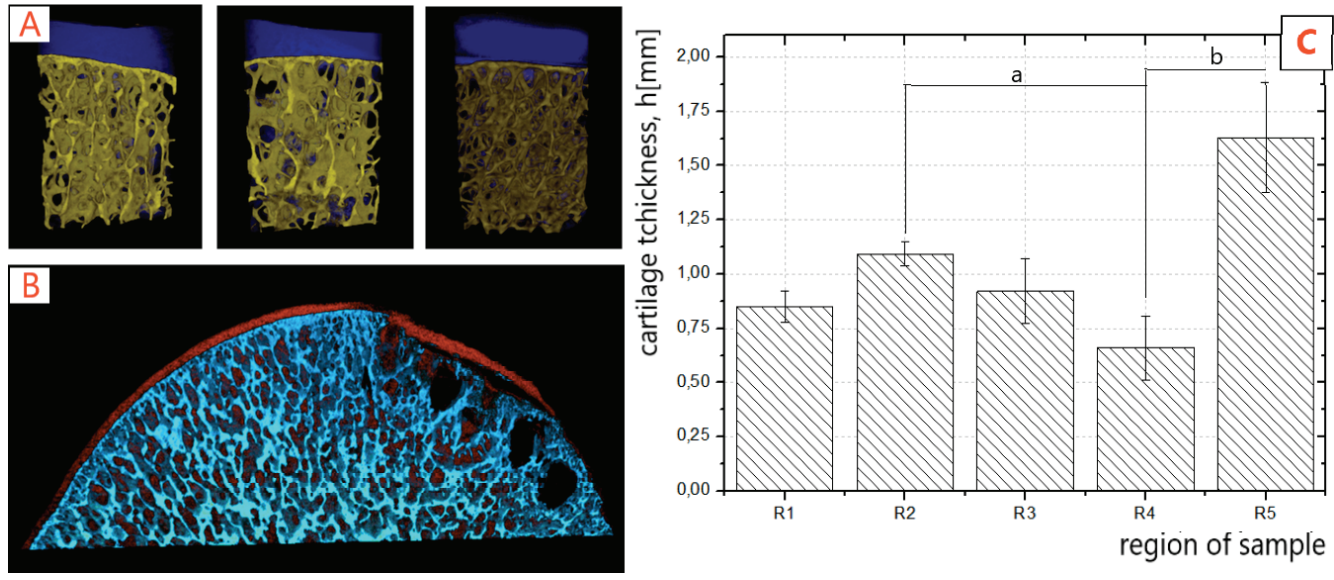

Fig. 4. Images of R1, R3 and R5 samples reconstructed from $\mu \mathrm{CT}$ images (A), and the intersection of the femur head with articular tissue mared in red (B).

Cartilage thickness for R1-R5 samples of all femur heads under investigation (C), $a, b$ significance at $p<0.05$ level

Sample reconstruction images are presented in Fig. 4A where cartilage tissue and the bone are marked with blue and yellow color respectively.

In Figure 4C and Table 1, cartilage thickness for different regions $\mathrm{R} 1-\mathrm{R} 5$ is presented. The thickness varied from 0.72 to $2.4 \mathrm{~mm}$ with samples from R5 region (anterior area of the femur bone) having the largest values and R4 (central area) having the smallest values. In R4 region, the changes of the tissue, due to coxartrisis, were the most clearly visible. These observations are inline with the results presented in the literature [13], [23]. Results for regions R2 and R4, as R5 and $\mathrm{R} 4$ are statistically significant with $p<0.05$.

\subsection{Histology of cartilage}

Histological evaluation were conducted on $0.5 \mathrm{~mm}$ thick tissue intersections extracted from three regions of the femur head (Fig. 1A). Histological analysis of articular cartilage at R1-R5 regions and compared to a control sample of normal cartilage (Fig. 5F). Morphological tests confirmed the changes observed in the $\mu \mathrm{CT}$ examination. The strongest reduction of the articular cartilage tissue was observed at R4, which was accompanied by an unformed mass of cartilage residue originating from the abraded surface and bursa fluid. Bone tissue in direct contact with articular cartilage has been significantly reduced. These changes correspond to stage 2 and 3, according to OARSI 1-6 stages, histologic features [0]. For the samples from R5 region the strongest thickening of the articular cartilage was observed, which may be caused by the two factors observed during the preparation: enhanced synthesis of the matrix by clearly enlarged chondrocytes, or shifts of the adaptive part of cartilage from the less loaded areas towards area with higher load. This can be demonstrated by the random distribution 
of chondrocytes throughout the cartilage and clear folding of the cartilage surface. Similar but less severe changes were seen in $\mathrm{R} 2$ point (posterior area). At R1, a slight thinning of the cartilage thickness was observed compared to the control sample, the bone bars are in the correct arrangement. In the R3 area (posterior area), structurally closest to the normal cartilage, areas with increased calcium ion saturation and slight thickening of bone trabeculae were observed (Fig. 5).
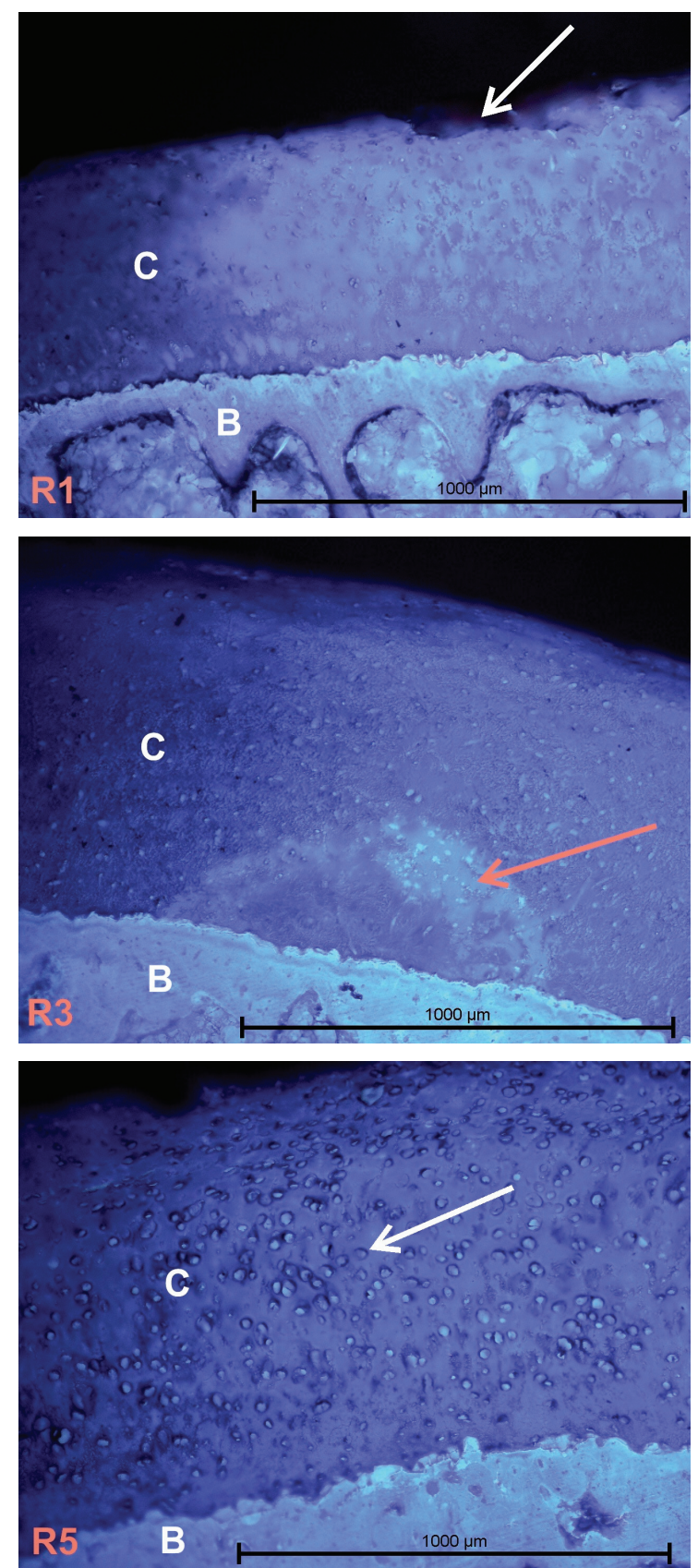

\subsection{Mechanical testing - indentation test}

Each cylindrical sample of $9.7 \mathrm{~mm}$ diameter was indented in four test points. The size of the samples and distances between the test points were large enough to ensure that individual indentation tests can be considered independent. Maximal indentation depth
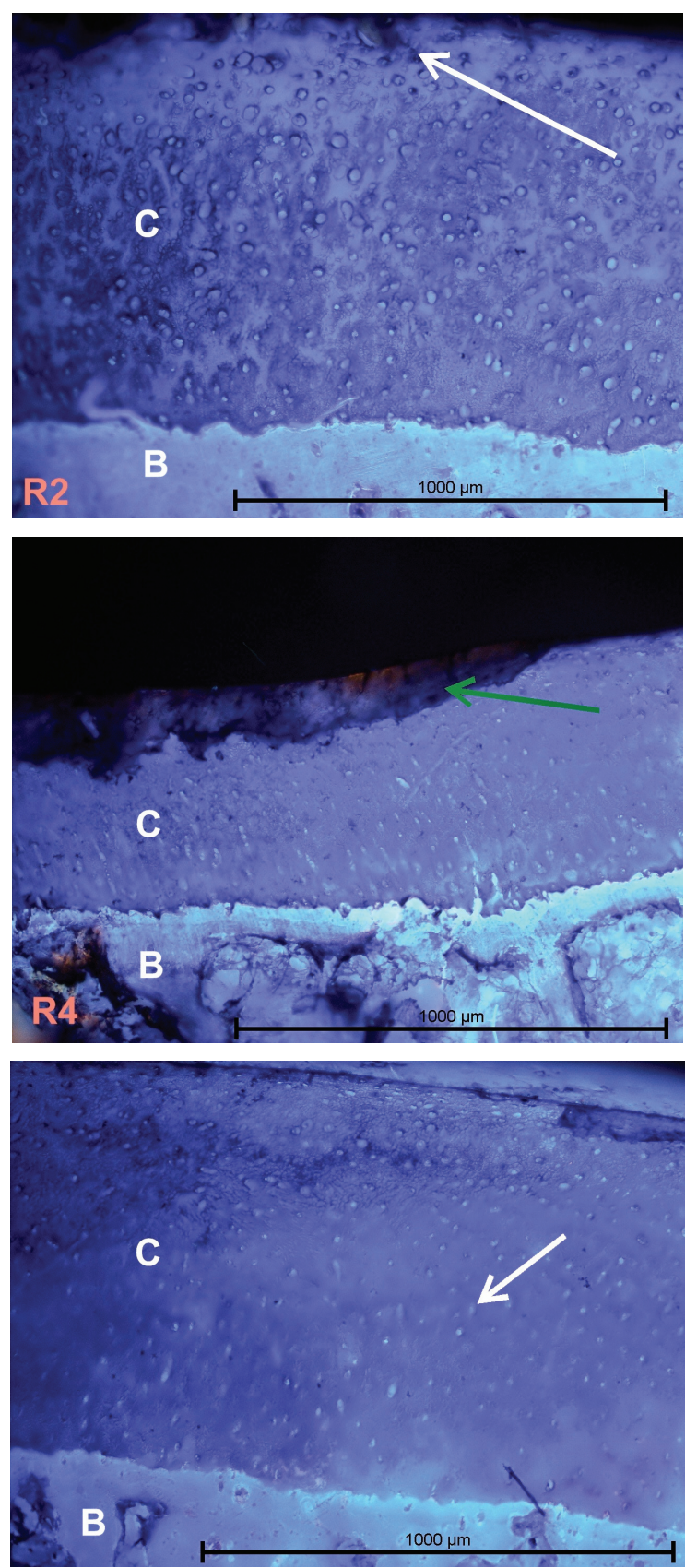

Fig. 5. Structure of the articular cartilage in analyzed areas: R1 - visible slight reduction of the cartilage thickness. The surface is partially damaged (arrow). Reduction in the chondrocyte size is noted; R2 - Large chondrocytes are visible throughout the entire cartilage. Rough surface (arrow) do not contain collagen fibers; R3 - regular structure of the cartilage is visible. In areas close to the bone the areas of calcifications are visible (arrow); R4 - Reduction in the cartilage thickness. The surface is covered by the remnants of degraded collagen fibers and products of fibrous capsula (green arrow); R5 - large, hypertrophied chondrocytes (arrow) present in the entire thickness of the cartilage; $\mathrm{C}$ - Normal cartilage with regular chondrocytes (arrow). Images taken with HiE, fluorescence, Mag 40× 

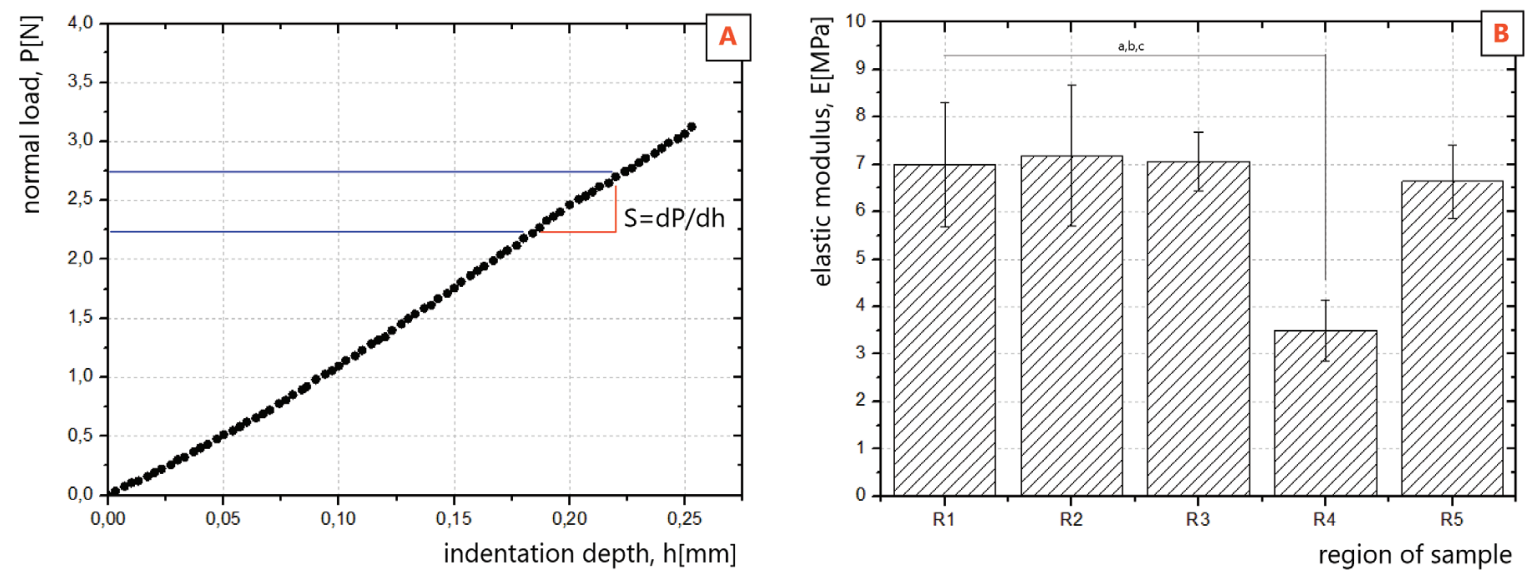

Fig. 6. A) Schematic representation of a load-indentation curve obtained from indentation test, to the maximal indentation depth of $0.25 \mathrm{~mm}$, MTS Synergie 100; B) Comparison of the elastic moduli $E$ obtained for different regions (R1-R5), a-d significance at $p<0.05$ level

was set to $0.25 \mathrm{~mm}$ and the tests points on the R1-R5 samples were selected so that the thickness of the cartilage in these points was at least $25 \%$ of maximal indentation depth (the effective value of a/h ratio varied between 12 to $32 \%$, Fig. 6) [21]. For this thickness, the value of $\omega / t$ was significantly larger than $0.1 \%$, which signals large strain of the samples. The value of $\kappa$ coefficient for the samples varied from 0.83 to 1.39 .

In Table 1, the mean values and standard deviations of mechanical properties such as: stiffness $(S)$, elastic modulus $(E)$, shear modulus $(G)$ and bulk modulus $(K)$, are shown. The results show that the difference between the mean values of $E, G$ and $K$ parameters for samples from different regions (R1-R3, R5 and R4) are significant. For the elastic modulus, the difference reaches $52 \%$ with the lowest $\mathrm{E}$ values for R4 (3.5 MPa) and the largest for R2 (7.2 MPa). However, the differences between the groups R1, R2, R3 and R5 are not significant. It can, therefore, be concluded that in the case of samples from these areas, the values of mechanical parameters are similar. For shear and bulk modulus the differences are close to $20 \%$, with the lowest values for R4 and the highest for $\mathrm{R} 2$ regions.

The $\mathrm{R} 2$ region had the maximum shear modulus (Table 1), while the lowest was in the R4 region. Regions R1, R2, R3 and R5 had similar shear modulus values, with a maximum difference of $7.5 \%$ between them. The largest bulk module (23.93 MPa) was present in the $\mathrm{R} 2$ region (Table 1), while in the $\mathrm{R} 4$ region the smallest bulk module (11.6 MPa) was found. These results were similar to those obtained for the shear modulus. Regions R1, R2, R3 and R5 showed values with a maximum difference of $7.5 \%$ between them.
Stiffness of the cartilage was calculated from $P$ and $\omega$ parameters estimated from the load-indentation curve. Samples from R2 and R3 regions have the largest stiffness (11.2 and $11.95 \mathrm{~N} / \mathrm{mm}$ ) while R4 (central area) samples have the lowest stiffness $(3.82 \mathrm{~N} / \mathrm{mm})$. Due to the large variation in the results for different specimens, the differences between different regions were statistically significant with $p>0.75$.

Table 1. Mechanical properties for different regions of the femur head form patients with advanced osteoarthrosis; $a-c$ significance at $p<0.05$ level

\begin{tabular}{|c|l|c|c|c|c|c|}
\hline $\begin{array}{c}\text { Test } \\
\text { region }\end{array}$ & $N=4$ & $\begin{array}{c}\text { Stiffness } \\
S[\mathrm{~N} / \mathrm{mm}]\end{array}$ & $\begin{array}{c}\text { Elastic } \\
\text { modulus } \\
E[\mathrm{MPa}]\end{array}$ & $\begin{array}{c}\text { Shear } \\
\text { modulus } \\
G[\mathrm{MPa}]\end{array}$ & $\begin{array}{c}\text { Bulk } \\
\text { modulus } \\
K[\mathrm{MPa}]\end{array}$ & $\begin{array}{c}\text { Thickness } \\
h[\mathrm{~mm}]\end{array}$ \\
\hline R1 & Mean & $10.266^{\mathrm{a}}$ & $6.997^{\mathrm{a}}$ & $2.413^{\mathrm{a}}$ & $23.324^{\mathrm{a}}$ & 0.848 \\
\hline & SD & 3.207 & 1.317 & 0.454 & 4.391 & 0.080 \\
\hline R2 & Mean & $11.263^{\mathrm{b}}$ & $7.178^{\mathrm{b}}$ & $2.475^{\mathrm{b}}$ & $23.927^{\mathrm{b}}$ & $1.093^{\mathrm{a}}$ \\
\hline & SD & 2.751 & 1.486 & 0.512 & 4.954 & 0.057 \\
\hline R3 & Mean & $11.947^{\mathrm{c}}$ & $7.061^{\mathrm{c}}$ & $2.435^{\mathrm{c}}$ & $23.536^{\mathrm{c}}$ & 0.920 \\
\hline & SD & 3.894 & 0.625 & 0.216 & 2.084 & 0.155 \\
\hline R4 & Mean & $3.842^{\mathrm{a}, \mathrm{b}, \mathrm{c}}$ & $3.495^{\mathrm{a}, \mathrm{b}, \mathrm{c}}$ & $1.205^{\mathrm{a}, \mathrm{b}, \mathrm{c}}$ & $11.648^{\mathrm{a}, \mathrm{b}, \mathrm{c}}$ & $0.794^{\mathrm{a}, \mathrm{b}}$ \\
\hline & SD & 0.938 & 0.648 & 0.224 & 2.161 & 0.191 \\
\hline R5 & Mean & 6.355 & 6.635 & 2.288 & 22.118 & $1.763^{\mathrm{b}}$ \\
\hline & SD & 1.452 & 0.772 & 0.266 & 2.573 & 0.297 \\
\hline
\end{tabular}

\section{Discussion}

Experimental investigations aiming to determine mechanical properties of cartilage tissue are challenging and complex. The results are affected by the heterogeneous properties of the tissue investigation method and its parameters, as well as conditions during sample preparation and tests. These include sam- 
ple hydration, area of the femur head from where it was taken, type of the test or load velocity. Indentation test is one of the most frequently used and enables the researcher to measure tissue mechanical properties in precisely selected location of the sample. Indentation also allows for the analysis of mechanical properties changes caused by the disease and can, therefore, provide information on the disease progress. The advantages of the method led to the increased number of investigation and research articles reporting information of the cartilage mechanical properties. However, most frequently, the research address cartilage of animal origin [15], [23] and less works report results for human cartilage from knee joint [7], [27] and hip [20]. Our article supplements previous works and provides more insight into mechanical properties of the femur cartilage.

The smallest values of the mechanical parameters were measured for samples from R4 region. Average value of the elastic modulus for R1, R2, R3 and R5 regions is equal to $6.8 \mathrm{MPa}$ while for $\mathrm{R} 4$ region it equals 3.9 MPa. Similar investigations were previously conducted by Richard [20], who measured mechanical properties of the cartilage after alloplastic surgery and the healthy cartilage. They have prepared samples from 4 different regions of the femur head (anterior, superior, posterior and inferior). The average values for the instantaneous elastic modulus of the healthy cartilage was equal to $10.9 \mathrm{MPa}$ while for OA tissues - to $3.4 \mathrm{MPa}$. The difference between our and Richard's results are close to $50 \%$, which might be due to the fact that Richard conducted experiments on cartilage in the advanced stage of the osteoarthritis. The conditions of the tissue made it difficult to prepare samples for the tests. Nevertheless, both research confirm that differences between the healthy and disease modified cartilage are significant and distinctive.

In our investigations, the material parameters $(E, G$, and $K$ ) were estimated using relations proposed by Hayes et al. [5] (Eq. (1)-(3)). These equations take into account $\kappa$ coefficient which is assumed to make the mechanical parameters independent of the sample thickness. This assumption, however, holds true only when the cartilage tissue has the same quality across the whole sample. For osteoarthritis, especially for femur head cartilage, the cartilage thickness changes and modifies its internal structure. This is a result of the change in the number and the structure of the collagen fibers inside the cartilage as well as the quality and location of the proteoglycans that sorb water and synovial fluid. These changes affect tissue elasticity and were observed in the histological investigations presented in our paper.
Similar values of the mechanical properties for R1, R2, R3 and R5 regions verify the assumption taken by Athanasiou [1], which states that the mechanical properties do not depend on the tissue thickness. Our results show that R5 region (part of superior area) has the biggest thickness that exceeds $2 \mathrm{~mm}$. The whole superior area has larger thickness compared to the other areas of the pathological tissue as well as normal tissue. The changes are clearly visible in the late stages of the osteoarthritis when the cartilage tissue thins in the areas of the largest load and the femur head deforms permanently. In the late stages of the disease, some areas of the femur head (especially the superior area) thicken.

For the central area of the femur head (R4 area) both the values of the mechanical parameters and the thickness of the cartilage were the smallest. The thickness was significantly reduced (approximately $50 \%$ compared to the R5 area), which might be the result of the changes in the load bearing in the cartilage/bone system. Decrease in the mechanical parameters in this area might result from the increased pressure in the most loaded areas or quite the opposite - decrease in the mechanical properties leads to loss of the cartilage and increases the pressure in the area.

Cartilage tissue is a viscoelastic material and behaves similarly to a sponge, absorbing and releasing synovial fluid when loaded and relieved. This behaviour not only changes the friction forces in the joint but is also responsible for transmission of nutrients the tissue cells. This is crucial function as cartilage tissue has no blood vessels. Ability to absorb the synovial fluid without losing the shape and mechanical properties is an important property of the cartilage as it ensures appropriate operation of the joint. When mechanical properties change the absorption and release processes are disrupted as a result of the changes in its permeability. This may reduce the amount of nutrients delivered to the tissue cells. In the late stages of the osteoarthritis, both the cartilage and the neighbouring bone tissue change. This leads to the changes in the biomechanical conditions in the cartilage/bone system and further deepens the changes in the subchondral tissue, including increased density and creation of cysts.

The above observations support the hypothesis that extensive tinning of the cartilage tissue leads to its reorganization and the decrease in the stiffness is an adaptation mechanism allowing the tissue to function in the changed load conditions. The exact and detailed description of the mechanisms of osteoarthritis development has not been discovered yet. It is, however, crucial to understand how the disease develops 
and how it would be possible to prevent it or reduce long-term disability.

The presented study has been conducted in a specific condition that may be seen as limitation. Because cartilage is a viscoelastic tissue, its properties significantly depend on the amount of water content. Consequently, it is important to maintain appropriate hydration of the sample during the whole investigation. This can be achieved through dipping the samples in isotonic PBS solution simultaneously, preventing the samples from excessive swelling [21].

Spherical shape of femur head makes it difficult prepare samples with surfaces that are parallel to the direction of the indentation. The use of small diameter indenter should minimize resulting inaccuracies.

\section{Conflict of interests}

The authors declare that there are no financial or personal relationships with other persons or organizations that could inappropriately influence (bias) this work.

\section{Acknowledgements}

This study was funded by the National Science Center grant number N N518 505139.

\section{Reference}

[1] Athanasiou K.A., Rosenwasser M.P., Buckwalter J.A., MaLinIN T.I., Mow V.C., Interspecies comparisons of in situ intrinsic mechanical properties of distal femoral cartilage, J. Orthop. Res., 1991, 9, 330-340.

[2] BADER D.L., BOWKeR P., Mechanical characteristics of skin and underlying tissues in vivo, Biomaterials, 1983, 305-308.

[3] Boughton O.R., Ma S., Zhao S., Arnold M., Lewis A., Hansen U., Cobb J.P., Giuliani F., Abel R.L., Measuring bone stiffness using spherical indentation, PLoS One, 2018, 12, 13 (7), e0200475.

[4] Halloran J.P., Sibole S., Van Donkelaar C.C., Van Turnhout M.C., Oomens C.W., Weiss J.A., Guilak F., ERDEMIR A., Multiscale mechanics of articular cartilage: potentials and challenges of coupling musculoskeletal, joint and microscale computational models, Ann. Biomed. Eng., 2012, 40 (11), 2456-2474.

[5] Hayes W.C., Keer L.M., Herrmann G., Mockros L.F., A mathematical analysis for indentation tests of articular cartilage, J. Biomech., 1972, 5 (5), 541-551.

[6] JIN H., Lewis J.L., Determination of Poisson's ratio of articular cartilage by indentation using different-sized indenters, J. Biomech. Eng., 2004, 126 (2), 138-145.

[7] Juras V.1., BitTsansky M., Majdisova Z., Szomolanyi P., Sulzbacher I., Gäbler S., Stampfl J., Schüller G., TRATTNIG S., In vitro determination of biomechanical properties of human articular cartilage in osteoarthritis using multiparametric MRI, J. Magn. Reson., 2009, 197 (1), 40-47.
[8] Keuttner K., Goldberg V.M., Osteoarthritic Disorders, American Academy of Orthopaedic Surgeons, Rosemont, 1995, XXI-XXV.

[9] Knecht S., Vanwanseele B., Stüssi E., A review on the mechanical quality of articular cartilage - implications for the diagnosis of osteoarthritis, Clin. Biomech., 2006, 21 (10), 999-1012.

[10] Kot M., Kobielarz M., Maksymowicz K., Assessment of mechanical properties of arterial calcium deposition, Transactions of Famena, 2011, 35 (3), 49-56.

[11] Krishnan R., Park S., Eckstein F., Ateshian G.A., Inhomogeneous cartilage properties enhance superficial interstitial fluid support and frictional properties, but do not provide a homogeneous state of stress, J. Biomech. Eng., 2003, 125 (5), 569-577.

[12] Kulej M., Dragan S., Kuryszko J., Kuropka P., WiduchowsKi W., Dragan S., Micromorphological assessment of bone tissue remodeling in various hip degeneration conditions, Adv. Clin. Exp. Med., 2020, 29 (1), 51-61.

[13] KuRRAT H.J., OBerländer W., The thickness of the cartilage in the hip joint, J. Anat., 1978, 126 (Pt 1), 145-155.

[14] Mankin H.J., Mow V.C., Buckwalter J.A., IANNotTi J.P., Form and function of articular cartilage, [in:] S.R. Simon (Ed.), Orthopaedic Basic Science, Rosemont, IL, American Academy of Orthopaedic Surgeons, 1994, 1-44.

[15] Mow V.C., GibBS M.C., LAI W.M., ZHU W.B., ATHANASIOU K.A., Biphasic indentation of articular cartilage - II. A numerical algorithm and an experimental study, J. Biomech., 1989, 22 (8-9), 853-861.

[16] Nevitt M.C., Lane N.E., Scott J.C., Hochberg M.C., Pressman A.R., Genant H.K., Cummings S.R., Radiographic osteoarthritis of the hip and bone mineral density. The Study of Osteoporotic Fractures Research Group, Arthritis Rheum., 1995, 38 (7), 907-916.

[17] Niederauer G.G., Niederauer G.M., Cullen L.C., JR., Athanasiou K.A., Thomas J.B., Niederauer M.Q., Correlation of cartilage stiffness to thickness and level of degeneration using a handheld indentation probe, Ann. Biomed. Eng., 2004, 32 (3), 352-359.

[18] Nikodem A., ŚcigaŁa K., Principles of osteoarthritis - its definition, character, derivation and modality-related recognition, InTech Europe Rijeka, Croatia, 2012, ISBN: 978-953$-51-0063-8$.

[19] NiKodem A., Correlations between structural and mechanical properties of human trabecular femur bone, Acta Bioeng. Biomech., 2012, 14 (2), 37-46.

[20] Richard F., Villars M., THIBAUd S., Viscoelastic modeling and quantitative experimental characterization of normal and osteoarthritic human articular cartilage using indentation, J. Mech. Behav. Biomed. Mater., 2013, 24, 41-52.

[21] Sophia Fox A.J., Bedi A., Rodeo S.A., The basic science of articular cartilage: structure, composition, and function, Sports Health., 2009, 1 (6), 461-468.

[22] Stempin R., Dragan S.Ł., Kulej M., Filipiak J., Dragan S., Effect of elliptical deformation of the acetabulum on the stress distribution in the components of hip resurfacing surgery, Acta Bioeng. Biomech., 2017, 19 (4), 35-41.

[23] Stolz M., Raiteri R., Daniels A.U., Van Landingham M.R., BASChONG W., AEBI U., Dynamic elastic modulus of porcine articular cartilage determined at two different levels of tissue organization by indentation-type atomic force microscopy, Biophys. J. 2004, 86 (5), 3269-3283. 
[24] TOMANIK M., NiKodem A., FILIPIAK J., Microhardness of human cancellous bone tissue in progressive hip osteoarthritis, J. Mech. Behav. Biomed. Mater., 2016, 64, 86-93.

[25] VAnNah W.M., Childress D.S., Indentor tests and finite element modeling of bulk muscular tissue in vivo, J. Rehabil. Res. Dev., 1996, 33 (3), 239-252.

[26] Venturato C., Pavan P.G., Forestiero A., Carniel E.L., NATALi A.N., Investigation of the biomechanical behaviour of articular cartilage in hindfoot joints, Acta Bioeng. Biomech., 2014, 16 (2), 57-65.

[27] Vidal-Lesso A., Ledesma-Orozco E., Daza-Benítez L., LESSO-ARROYO R., Mechanical characterization of femoral cartilage under unicompartimental osteoarthritis, Ingenieria Mecanica, 2014, 4 (6), 239-246.
[28] Vignon E., Arlot M., Meunier P., Vignon G., Quantitative histological changes in osteoarthritic hip cartilage: morphometric analysis of 29 osteoarthritic and 26 normal human femoral heads, Clinical Orthopaedics and Related Research, 1974, 103, 269-278.

[29] Wojtkow M., Kielbowicz Z., Biezynski J., Pezowicz C., Quantitative and qualitative assessment of the impact of osteoporosis on endplate layers, Biocybern. Biomed. Eng., 2019, 39, 797-805.

[30] Zhang M., Zheng Y.P., MAK A.F., Estimating the effective Young's modulus of soft tissues from indentation tests - nonlinear finite element analysis of effects of friction and large deformation, Med. Eng. Phys., 1997, 19 (6), 512-517. 